\title{
Charmonium resonance production from quark coalescence
}

\author{
Gergő Hamar* \\ MTA KFKI RMKI Research Institute for Particle and Nuclear Physics, \\ Budapest, Hungary \\ E-mail: hamargergodrmki.kfki.hu

\section{Péter Lévai} \\ MTA KFKI RMKI Research Institute for Particle and Nuclear Physics, \\ Budapest, Hungary \\ E-mail: plevaiermki.kfki.hu
}

We have considered a relativistically invariant quark coalescence model to predict hadronic resonance productions in heavy ion collisions. We extended our model - which has been applied earlier for strange and non-strange hadrons - to describe charmonium meson ratios, namely $J / \psi$, $\Psi^{\prime}$ and $\chi_{c}$, at RHIC energies. In the applied quark coalescence model the width of the produced mesons and resonances plays an important role and determines the yield of these particles. In the charm sector the values of meson width have a special structure, which feature enhances the importance of meson width in the population of the different charmonia channels. We display our numerical results at RHIC energies.

European Physical Society Europhysics Conference on High Energy Physics, EPS-HEP 2009,

July 16 - 222009

Krakow, Poland

*Speaker. 


\section{Motivation, coalescence models}

Proper particle detection methods gave us the possibility to study resonance and charmonia productions in heavy ion collisions. The measured resonance yields at RHIC and even at SPS energies differ from the thermal expectations [i].

The quark number scaling of the asymmetric flow $v_{2}$ [2] cannot be interpreted in thermal models, however it is a standard consequence of the coalescence type hadronizations. Quark coalescence models succeed not only at the prediction of particle yields, but they can also describe the pion to proton anomalous ratio.

The first quark coalescence model, ALCOR [B], made to describe the hadronization of a hot quark matter, can predict the particle yield ratios. Hadron spectra have been constructed in the MICOR model [4]. TThese quantum mechanics-based statistical methods appear to be efficient and robust in predicting the final hadron yields. [5]. From then on, new coalescence and recombination models have been born, and have been applied successfully to RHIC data.

We introduce a relativistic resonance coalescence model, and show the calculated light, strange and charm quark yields for RHIC energies [6]. Charm sector can be inserted into this model and the charmonia production can be studied.

\section{Resonance coalescence}

In the former coalescence models the primary prehadrons have been identified directly with elements of the stable or the first excited hadron multiplets. Hadrons are characterized by a sharp, Dirac-delta type mass spectra, although the resonances with final widths are known (e.g. $\rho(770)$, $K^{*}(892)$ ). High mass resonances cannot be produced with these methods.

Introducing heavier resonances generates unexpected problems: a) The mass of a prehadron is the sum of the constituent valence quark masses. b) Resonances with higher mass will dominate a hadronization channel.

Since experimental data are available for many resonances, thus we have included them in the calculation within the improved quark-coalescence model.
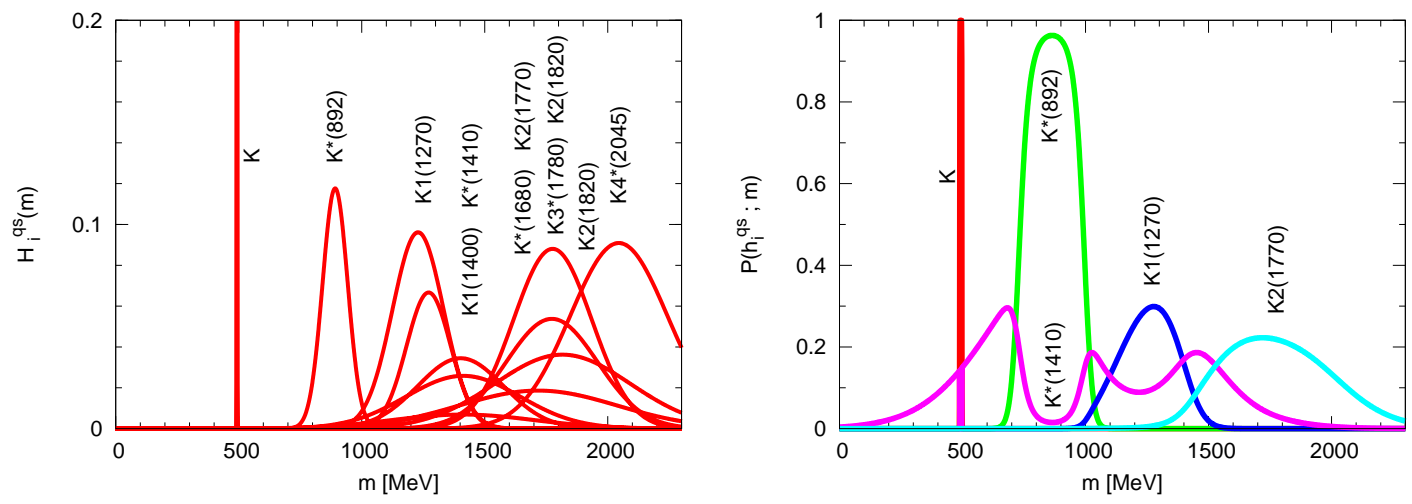

Figure 1: The hadronic spectral functions, $H_{i}^{Q}(m)$, with quantum numbers $Q=q \bar{s}$ (left panel). The probability functions, $\mathscr{P}\left(h_{i}^{Q} ; m\right)$, for five strange mesons with quantum numbers $Q=q \bar{s}$ (right panel). Note the special structure of $P\left(K^{*}(1410) ; m\right)$ (magenta line). 
To avoid the former problems in our relativistic resonance coalescence model [6] we create prehadrons with a continuous mass spectra, which will be determined from relativistic kinematics:

$$
m_{\text {prehadron }}=M_{q \bar{q}}=\left\|p_{q_{1}}^{\mu}+p_{q_{2}}^{\mu}\right\|=\sqrt{\left(E_{1}+E_{2}\right)^{2}-\left(\overrightarrow{p_{1}}+\overrightarrow{p_{2}}\right)^{2}} .
$$

This way one can obtain arbitrarly large values for $m_{\text {prehadron }}$, thus all the heavier resonances become accessible. Chosen the initial quark momentum distributions the invariant mass spectrum, $J^{Q}(m)$, is constructed for prehadrons characterized by quantum numbers $Q$ as

$$
J^{Q}(m)=\int_{0}^{\infty} \int_{0}^{\infty} d^{3} \vec{p}_{1} d^{3} \vec{p}_{2} f\left(m_{1}, \vec{p}_{1}\right) f\left(m_{2}, \vec{p}_{2}\right) \delta\left(m-\left\|p_{1}^{\mu}+p_{2}^{\mu}\right\|\right) .
$$

We used the relativistic Jüttner distribution for the light and strange quarks (anti-quarks) with the same temperature parameter $(T=180 \mathrm{MeV})$. The shape of these mass distributions (see Figure 2 for charm) give an exponential-like suppression for the heavier resonances.

One should find the way to connect the continuous function of the invariant mass spectrum, $J^{Q}(m)$, to the mass spectra of the existing hadron resonances . Considering $h_{i}^{Q}$ hadron resonance with quantum numbers $Q$, knowing its mass, width and degeneracy we can construct its spectral function $H_{i}^{Q}(m)$ by the usual Breit-Wigner formula (see Figure 1 left panel).

The probability to produce resonance $h_{i}^{Q}$ from a given prehadron mass $m$ with the same quantum numbers can be defined as a relative weight of its spectral function at $m$ compared to the sum of all the spectral functions at $m$. Figure 1 (right panel) displays this $\mathscr{P}\left(h_{i}^{Q} ; m\right)$ for few $Q=q \bar{s}$ resonances.

The production rate is given by the combination of probabilities and proper mass spectra. The primary yield is generated similarly to the usual coalescence models.

\section{Charmonia production}

One can insert new quark flavours into the model, by giving the effective quark mass and its momentum distribution. For the charm quarks let us take the usual Jüttner distribution with the same temperature parameter as for the light quarks before. Choosing an effective charm mass one can generate the invariant mass distributions, the appearance functions and the yields. Varying the two parameters, mass and temperature (Fig. 2. left) one can get interesting effects.
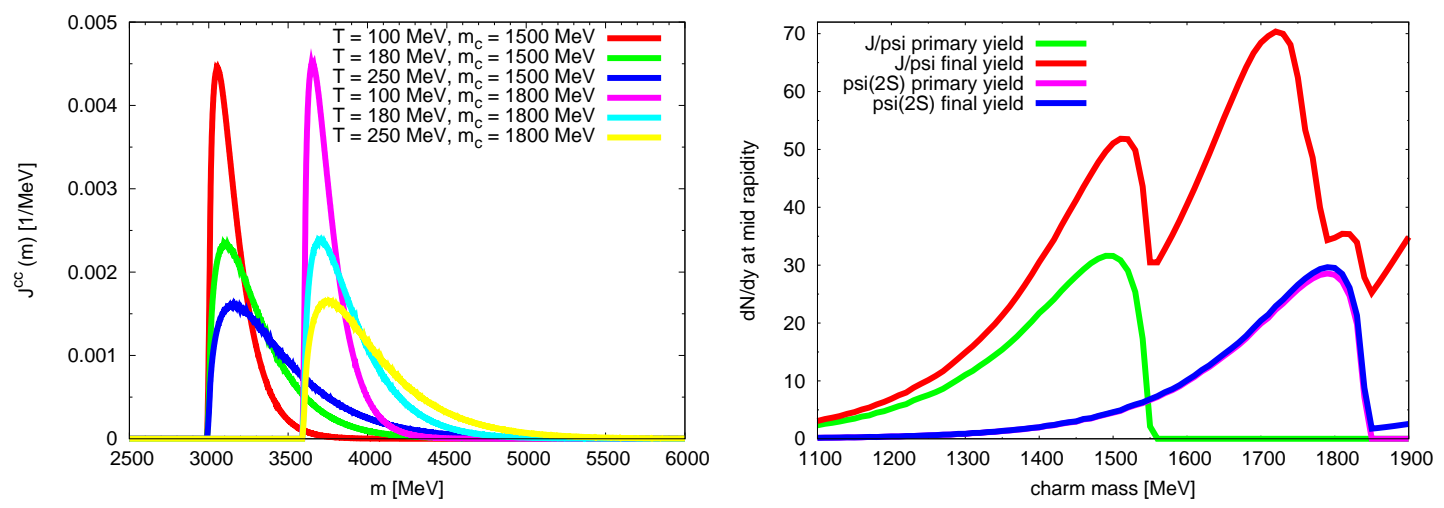

Figure 2: (Left) Pre-charmonia invariant mass distribution with different charm mass and temperature parameters of the initial Jüttner momentum distributions. (Right) Primary and final yields of two charmonium states depending on the initial choice of the effective charm mass. 

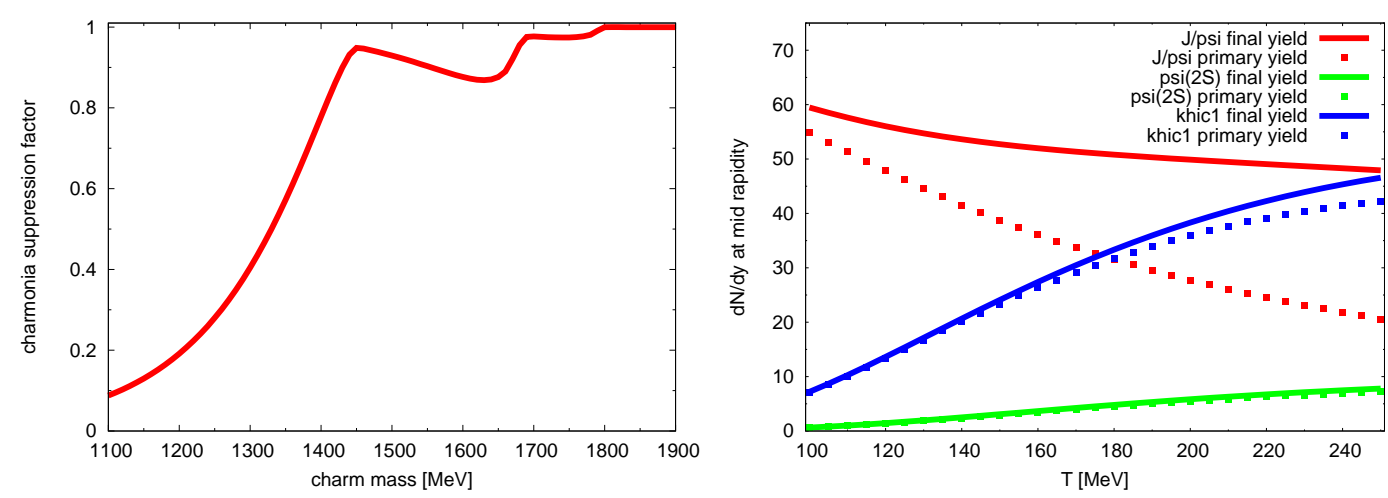

Figure 3: (Left) Charmonia suppression factor shows that light charm mass prefers charm meson over charmonium states production. (Right) Primary and final yields depending on the temperature. High temperature relatively increases the primary production of higher mass states. $J / \Psi$ final yield is not sensitive to the temperature due to its feed from the decay chains of the heavier charmonium states.

Charmonia masses have a special structure, namely the high mass region is densely covered by the resonance states, however there are few sharp states in the light mass region (less than $3.2 \mathrm{GeV}$ ). This causes a strong dependence of the primary yields on the choice of the introduced effective charm mass. States with mass smaller than $2 m_{\text {charm }}$ can be produced only through decay chains. On the right side of figure 2 we show the primary and final yields of some well measurable charmonium states, where this effect can be seen.

Due to the mass state structure of charmonia the effective charm mass influences the ratio of charm quarks hadronizing into charm mesons versus charmonia. This can be introduced as a charmonia suppression factor, which equals unity at heavy $m_{\text {charm }}$ and is smaller than one in the light-mass region (Fig. B).

On the left side of figure 2 one can see the extension of the invariant mass distributions to higher masses as the temperature rises. Therefore the higher the temperature the more likely heavy charmonium states are produced. Accidentally $J / \Psi$ final yield is not effected by the temperature, because it gets feed-down from the heavy charmonia decays.

This work has been supported by the Hungarian OTKA grants No. NK062044, H07-C 74164.

\section{References}

[1] P. Seyboth, Recent results from nucleus-nucleus collisions at the CERN SPS, J. Phys. G 35104008

[2] D. Molnár, S. A. Voloshin Elliptic flow at large transverse momenta from quark coalescence Phys. Rev. Lett. 91092301

[3] T. S. Biró, P. Lévai, J. Zimányi ALCOR: A Dynamic model for hadronization Phys. Lett. B 3476

[4] P. Csizmadia, P. Lévai $\phi, \Omega$ and $\rho$ production from deconfined matter..., Phys. Rev. C 61031903

[5] G. Hamar, L. L. Zhu, P. Lévai, P. Csizmadia, The robustness of quasi-particle coalescence in quark matter, Eur. Phys. J. Special Topics 15567

[6] G. Hamar, P.Lévai Resonance production in a quark coalescence framework J. Phys. G 35104075 